\title{
The need for medical education reform: genomics and the changing nature of health information
}

\author{
Elizabeth A Nelson ${ }^{1}$ and Amy L McGuire*2
}

\begin{abstract}
No course in genetics can prepare the practicing physician to interpret whole-genome data. We argue that genetics is a microcosm of the changing dynamics of the practice of medicine. It illustrates the perfect storm of exponential increases in raw data with undetermined clinical relevance, ease of access to large amounts of data via the internet and shifting expectations of the doctor-patient relationship and the very mechanisms of health care delivery. Educational reform is needed across the continuum of medical education, from the student to the faculty training them, and requires a shift in focus from factual knowledge to data management and interpretation.
\end{abstract}

\section{Physician, patient and personalized medicine}

Most physicians would say that good medicine has always been personalized. Physicians use their medical expertise to apply known data to the lifestyle and health of the individual patients in their offices. Yet patients interested in the concept of 'personalized' medicine are no longer satisfied with a discussion that involves population-based benchmarks and generic side effect profiles.

Many believe that a physician's ability to provide more personalized information on the basis of a patient's individualized genetic and epigenetic profile will soon be a reality given the technological advances of the past decade and the unprecedented wealth of biological data that has been generated by the Human Genome Project. Yet studies suggest that most physicians do not have the expertise to interpret even the simplest of genetic tests [1]. To prepare physicians for the onslaught of genomewide information, some have suggested that courses in genetics be integrated throughout the entire medical

*Correspondence: amcguire@bcm.edu

${ }^{2}$ Center for Medical Ethics and Health Policy, Baylor College of Medicine, One

Baylor Plaza, Houston, Texas 77030, USA

Full list of author information is available at the end of the article school curriculum [2,3]. Although additional training in genetics may be necessary, we argue that it is not sufficient.

A single course cannot prepare the practicing physician sufficiently to interpret complex whole-genome data. As understanding of the functional significance of gene variants increases, automated systems that can provide updated clinical decision support to physicians will be essential [4]. The physician must learn to use the newest bioinformatic tools available to access interpretive information and to make judgments about appropriate follow-up treatment and care.

Physicians must also be prepared for the changing nature of the physician-patient relationship. As early as 1973 it was recognized that the doctor-patient relationship was changing, owing in part to 'a growing biomedical literacy and awareness among the patient population' [5]. The use of the internet and search engines is accelerating this change. Patients' access to information (genetic as well as generic health information) has increased substantially and is likely to continue to grow. The internet and direct-to-consumer marketing of sources of health information allow the patient to walk into some clinical encounters with as much, if not more, information than the physician. As the era of clinical genomics matures, the patient and the physician will be learning about this field and how it affects their health at the same time.

\section{Reform in medical education}

The dynamics of the practice of medicine are changing, and genetics is just one example of this. There has been an exponential increase in raw data that have undetermined clinical relevance and in the ease with which physicians and patients can access large amounts of data via the internet. The expectations of patients about the doctorpatient relationship and about the very mechanisms of health care delivery are also shifting. These changes, illustrated by genetics but also found in other areas, combine and interact to have an enormous effect on how physicians work. How we approach educational reform should not focus only on increasing knowledge but also on how physicians manage the amount of scientific data readily available both to them and to the public at large. 
How should we approach the shift in medical education? Since the Flexner Report in 1910 [6], several substantive reviews of US medical education have acknowledged that too much information is being given to medical students whereas, at the same time, there is a need to provide them with the latest information on medical discoveries $[7,8]$. In 2010, these educational tensions persist.

If medical knowledge doubles every 3 to 5 years, there is no way to teach a student every medical fact and relationship. Future physicians, more than ever before, must be able to retrieve and interpret data and to use and understand the significance of informatics, probabilities and decision-making assumptions. Medical students should also be taught to navigate and evaluate electronic resources. They should understand the research processes, the application of emerging information and how new knowledge is developed. Certainly the application of these skills requires a backbone of medical facts and relationships, but these facts are the vocabulary of medicine, the building blocks, not the final product.

\section{Who needs to learn these new skills: from pre-med to continuing medical education}

Reform is needed across the educational continuum, from premedical requirements all the way through to continuing medical education (CME). According to the American Association of Medical Colleges, a third of the students entering medical school have non-science/ mathematics backgrounds. They are required by US medical schools to have basic courses in inorganic and organic chemistry, biology, physics, English and calculus, but there are no requirements for statistics, ethics or computational coursework. Yet competencies such as critical thinking, statistical analysis and decision-making are essential to meet the challenges of the information explosion. The skill set of the entering medical student needs to be refined accordingly.

As current US medical students make the transition into their individual, departmental residency programs, collectively known as graduate medical education (GME), they have little foundation in how to apply emerging research (genomic or other) into clinical practice. The Accreditation Council for Graduate Medical Education (ACGME) competencies are the backbone of GME across departments. The competency of 'Practice Based Learning and Improvement' would lend itself to developing curricula that creates 'the experiential bridge between continuous learning and good patient care' [9]. There is an educational literature spanning a decade on the need for this, but little or no change has been documented.

The application of emerging research into clinical practice is not a new problem in the arena of the practicing physician either. Didactic CME courses have little data to support their ability to change clinical practice [10]. Re-certification requirements (usually based on hours of CME courses taken) focus on updating factual knowledge, not the ability to navigate emerging data.

There are programs in existence to retrain physicians who have left the workforce and want to re-enter. Similar programs could be developed to train the practicing physician in the evaluation and interpretation of emerging topics. We need to explore which training models work best, which produce an impact on clinical practice and how these outcomes could provide feedback to improve training at all levels.

Finally, academic faculties who teach medical education are struggling like the practicing physician to assimilate emerging data. Faculty development is essential to prepare academics who teach medical learners. This may be the greatest barrier to change. Fortunately, there are efforts under way both in the USA and internationally to bring together innovative thinkers in faculty development to explore this important issue. These include one recent and two upcoming conferences: 'A 2020 Vision of Faculty Development Across the Medical Education Continuum' in Houston, Texas, the Universitas 21st Annual Health Sciences Meeting in Monterrey, Mexico, and the 1st International Conference on Faculty Development in the Health Professions in Toronto, Canada.

\section{Conclusions}

The growing field of genomics provides the most visible example of the explosion of medical data, but it is still only one component of the rapidly changing face of modern health care.

We have been describing the need for educational reform long enough. The genotype has been sequenced but the phenotype of the educational modalities remains minimally expressed. The time has come to make changes not in the factual content of medical education but in the thinking process that physicians in this century will need to manage the unique challenges of the information explosion.

Future medical students will need to develop skills in manipulating data mining tools, evaluating data, problem solving and navigating the emerging health care delivery system. A system in which care will be delivered by interdisciplinary teams will require an integrated, 'personalized' treatment plan.

\section{Abbreviations}

CME, continuing medical education; GME, graduate medical education.

\section{Competing interests}

The authors declare that they have no competing interests.

\section{Authors' contributions}

AM and EN drafted and edited the article; AM contributed the genetic and ethics expertise and EN contributed the medical education expertise. 


\section{Author details}

'Undergraduate Medical Education, Baylor College of Medicine, One Baylor Plaza, Houston, Texas 77030, USA. ${ }^{2}$ Center for Medical Ethics and Health Policy, Baylor College of Medicine, One Baylor Plaza, Houston, Texas 77030, USA.

Published: 17 March 2010

\section{References}

1. Guttmacher AE, Porteous ME, Mclerney JD: Educating health care professionals about genetics and genomics. Nat Rev Genet 8:151.

2. Salari $\mathrm{K}$ : The dawning era of personalized medicine exposes a gap in medical education. PLoS Med 2009, 6:e1000138.

3. Avar D, Knoppers B: Genomic medicine: considerations for health professionals and the public. Genome Med 2009, 1:25.

4. McGuire AL, Belmont J: The futility of genomic counseling: Essential role of electronic health records. Genome Med 2009, 1:48.

5. A growth in biomedical literacy is changing the doctor-patient relationship. Current Contents 1973, 14:5-7. [http://www.garfield.library. upenn.edu/essays/V1 p425y1962-73.pdf]
6. Flexner A: Medical Education in the United States and Canada: a Report to the Carnegie Foundation for the Advancement of Teaching. New York: Carnegie Foundation for the Advancement of Teaching; 1910.

7. Rappleye WC: Medical education. J Higher Ed 1930, 1:154-159.

8. Coggeshall LT: Planning for Medical Progress through Education. Evanston: Association of American Medical Colleges; 1965.

9. Lynch DC, Swing SR, Horowitz SD, Holt K, Messer JV: Assessing practicebased learning and improvement. Teach Learn Med 2004, 16:85-92.

10. Davis D, O'Brien MA, Freemantle N, Wolf FM, Mazmanian P, Taylor-Vaisey A: Impact of formal continuing medical education: do conferences, workshops, rounds, and other traditional continuing education activities change physician behavior or health care outcomes? JAMA 1999, 282:867-874.

doi:10.1186/gm139

Cite this article as: Nelson EA, McGuire AL, et al.: The need for medical education reform: genomics and the changing nature of health information. Genome Medicine 2010, 2:18 